\title{
Supplementary Material: On Modeling the Southern Ocean Phytoplankton Functional Types
}

Svetlana N. Losa ${ }^{1,2}$, Stephanie Dutkiewicz ${ }^{3}$, Martin Losch ${ }^{1}$, Julia Oelker ${ }^{4}$, Mariana A. Soppa ${ }^{1}$, Scarlett Trimborn ${ }^{1}$, Hongyan $\mathrm{Xi}^{1}$, and Astrid Bracher ${ }^{1,4}$

${ }^{1}$ Alfred Wegener Institute Helmholtz Centre for Polar and Marine Research, Bremerhaven, Germany

${ }^{2}$ Shirshov Institute of Oceanology, Russian Academy of Sciences, Moscow, Russia

${ }^{3}$ Massachusetts Institute of Technology, Cambridge, Massachusetts, USA

${ }^{4}$ Institute of Environmental Physics (IUP), University of Bremen, Bremen, Germany

Correspondence: Svetlana Losa (Svetlana.Losa@awi.de)

\section{S1 Protocol and Summary of Prior Darwin Sensitivity Experiments}

We conducted a series of experiments with the diatoms size considered as $8 \mu m$ (default), $18 \mu m, 16 \mu m$ and $15 \mu m$ and, respectively, changed the growth rate and nutrients uptake (maximum photosynthesis rate $P_{\max }^{C}$ and nutrients half saturation rate $k_{s} a t$, see Tables S1 - S4, Ward et al. 2017). A more accurate timing of the Southern Ocean diatom blooms was obtained for the experiments with diatoms size being $18 \mu \mathrm{m}$. In this experiment, the spatial distribution of the phytoplankton dominance and diatoms Chla phenology also agreed better with the PFT dominance provided by PHYSAT satellite data product ( $h t t p$ : //log.cnrs.fr/Physat - 2?lang $=f r$ ) and satellite-based estimates of diatom phenological indices (Soppa et al., 2016). However, the quality of the simulated diatom phenology degraded at lower latitudes. Finally, two size classes of diatoms (large and small) have been introduced in the model, which led to further improvement of the model - observations agreement for the Southern Ocean PFT phenology and spatial distribution (for instance along the Western Antarctica Peninsula and in the Drake Passage, Trimborn et al. 2015.

\section{S1.1 Protocol}

Below we provide a detailed protocol of the sensitivity experiments. It is worth commenting that due to computing limitation these experiments were, sometimes, initialized at different time (but run over three - five model years), and the model outputs are considered and evaluated in a climatological mean context.

\section{S1.1.1 The task: to obtain plausible co-existence of haptophytes in the Southern Ocean}

Table S1 summarises the specified PFT traits. Initially, mortality of large phytoplankton was decreased by a factor of two in comparison with the default value $\left(0.1\right.$ day $\left.^{-1}\right)$ used in Dutkiewicz et al. (2015).

Additional changes: palatability factors for coccolithophores $\left(r_{j=6, k=1}\right)$, other large non-diatoms $\left(r_{j=2, k=1}\right)$ and $\mathrm{N}$-fixer. 
Table S1. Assumed size and photosynthesis parameters of the simulated PFTs

\begin{tabular}{lcccccc}
\hline Param $\backslash$ PFTs & diatom & Phaeo & small euk & Prochlor & Nfixer & coccolitho \\
\hline size $(d m)$ & 8 & 5 & 0.9 & 0.6 & 8 & 5 \\
$P_{\text {max }}^{C}$ & 2.42 & 1.67 & 1.15 & 1.09 & 0.31 & 1.34 \\
$k_{\text {sat }_{N}}$ & 0.239 & 0.110 & 0.030 & 0.007 & & 0.092 \\
$k_{\text {sat }_{P}}$ & 0.015 & 0.007 & 0.002 & 0.0004 & 0.0029 & 0.006 \\
mfunc & silicified & & & & & calcifier \\
\hline
\end{tabular}

with Phaeo for Phaeocystis-like ("other large"), small euk for small eukaryotes, Prochlor for Prochlorococcus, Nfixer for nitrogen fixing PFT and coccolith for coccolithophores
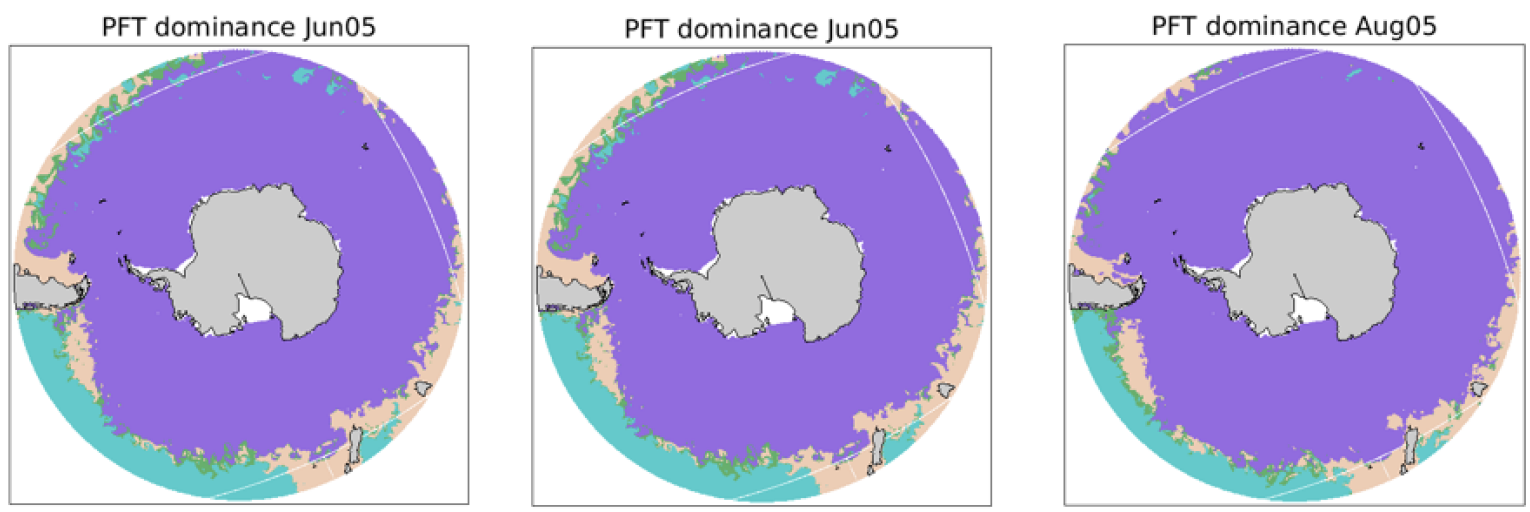

mixed diatom hapto pico

Figure S1. The Southern Ocean PFT dominance simulated with Darwin-MITgcm for June-July-August.

Results: in winter - early spring (June-July-August) diatoms distribute too much to the north (Figure S1); too early diatom bloom.

\section{S1.1.2 "Large diatoms" series}

The task: to test the hypothesis that the model deficiencies in correct representation of diatom phenology and dominance result

5 from the discrepancies between the assumed size of model diatom $(8 \mu \mathrm{m})$ and size of diatoms observed in the Southern Ocean (> $20 \mu m$, see Table S2)

Additional changes: palatability factors for coccolithophores $\left(r_{j=6, k=1}\right)$, other large non-diatoms $\left(r_{j=2, k=1}\right)$ and $\mathrm{N}$-fixer.

Results: diatom phenology is better simulated at high latitudes, however not at lower latitudes; other large eukaryotes are underestimated, while coccolithophores are highly overestimated (overgrow all other PFTs). The spatial distribution of small eukaryotes shows some patterns observed for Phaeocystis (Figure S2, central panel). The Southern Ocean phytoplankton 
Table S2. Assumed size and photosynthesis parameters of the simulated PFTs

\begin{tabular}{lcccccc}
\hline Param $\backslash$ PFTs & diatom & Phaeo & small euk & Prochlor & Nfixer & coccolitho \\
\hline size $(d m)$ & 18 & 5 & 2 & 0.6 & 11 & 5 \\
$P_{\max }^{C}$ & 1.79 & 1.67 & 1.15 & 1.09 & 0.30 & 1.34 \\
$k_{\text {sat }}$ & 0.451 & 0.11 & 0.03 & 0.007 & & 0.093 \\
$k_{\text {sat }_{P}}$ & 0.028 & 0.007 & 0.002 & 0.0004 & 0.036 & 0.006 \\
$r_{j, k=1}$ & & 0.79 & & & & \\
mfunc & silicified & & & & & calcifier \\
\hline
\end{tabular}

Nomenclature as in Table S1
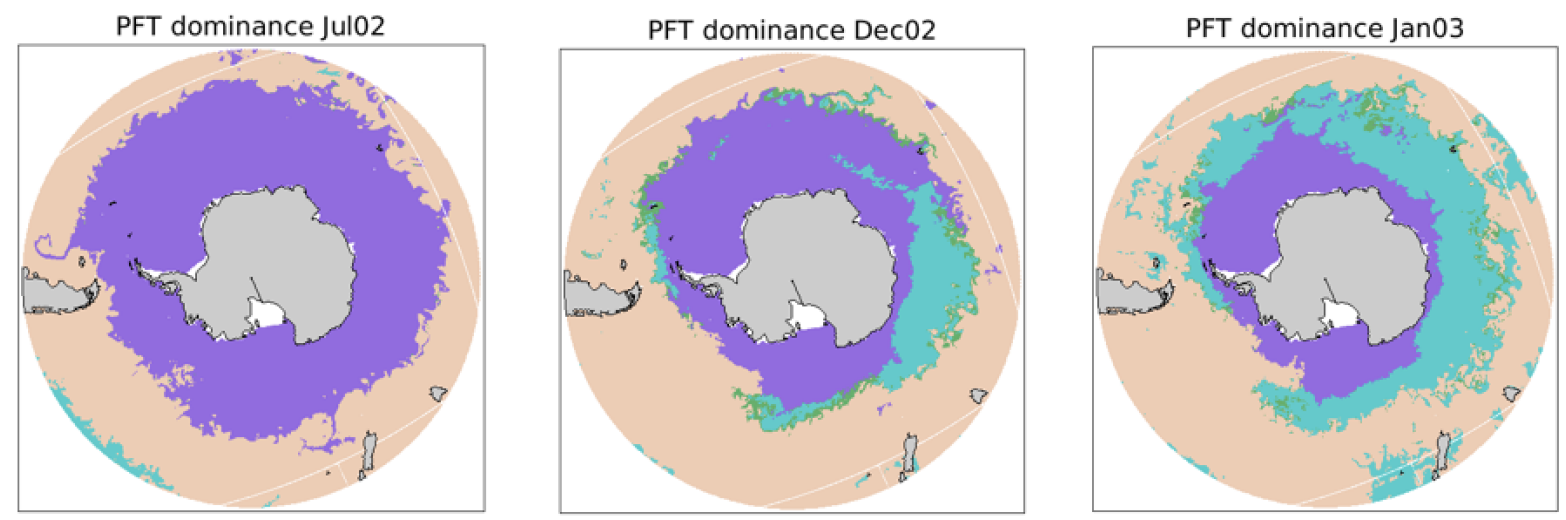

mixed diatom hapto pico

Figure S2. The Southern Ocean PFT dominance simulated with Darwin-MITgcm for July 2002, December 2002 and January 2003.

dominance (Figure S2) agrees better with PHYSAT satellite retrievals in comparison with results presented in the study by Dutkiewicz et al. (2015) However, simulated diatom blooms still start earlier in comparison with what is observed.

\section{S1.1.3 "Small eukaryotes" - not silicified, including Phaeocystis singular cell" series}

The main assumption: based on previous series, small eukaryotes ("other pico") represent Phaeocystis ant. solitory cells 5 (Table S3).

The task: to decrease the abundance of coccolithophores and improve Phaeocystis simulations; sensitivity to the size of large diatoms.

Changes relative to "Large diatoms": size of diatoms - $15 \mu \mathrm{m}$;

Results: distribution of pico eukaryotes reveals some features and patterns observed for Phaeocystis ant. (Alvain et al., 2008)

10 as before (Figure S3); "other large" (nano-) overcompete coccolithophores; 
Table S3. Assumed size and photosynthesis parameters of the simulated PFTs

\begin{tabular}{lcccccc}
\hline Param $\backslash$ PFTs & diatom & Phaeo & small euk & Prochlor & Nfixer & coccolitho \\
\hline size $(d m)$ & 15 & 5 & 2 & 0.6 & 11 & 5 \\
$P_{\text {max }}^{C}$ & 1.93 & 1.67 & 1.15 & 1.09 & 0.30 & 1.34 \\
$k_{\text {sat }_{N}}$ & 0.393 & 0.11 & 0.03 & 0.007 & & 0.093 \\
$k_{\text {sat }_{P}}$ & 0.025 & 0.007 & 0.002 & 0.0004 & 0.036 & 0.006 \\
mfunc & silicified & & & & & calcifier \\
\hline
\end{tabular}

Nomenclature as in Table S1
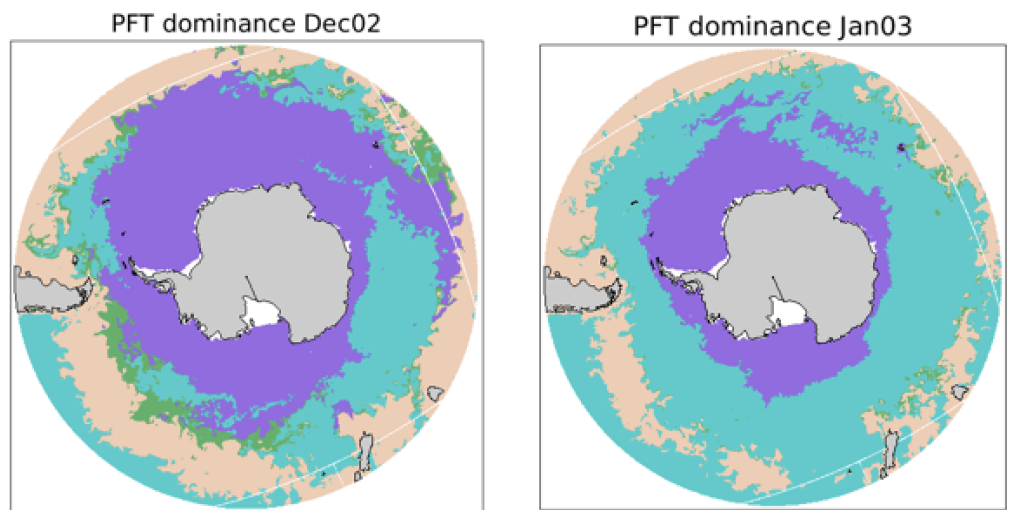

mixed diatom hapto pico

Figure S3. The Southern Ocean PFT dominance simulated with Darwin-MITgcm for December 2002 and January 2003.

Recommendations: to adjust mortality of coccolithophores (decrease); $r_{j=2, k=1}-0.79$; size of diatoms $-16 \mu m$ (or larger, later consider large and small diatoms!).

\section{S1.1.4 "Small eukaryotes" - diatoms series}

The main assumption: small eukaryotes ("other pico") represent Phaeocystic ant. solitary cells and small diatoms.

5 The task: to investigate whether the considering small diatoms would improve PFT phenology and composition at lower latitudes in the Southern Ocean.

Changes: size of large diatoms $-16 \mu \mathrm{m}$, size of smaller eukaryotes $-1.8 \mu \mathrm{m}$ but they include silicifiers (diatoms); Prochloroccus is not photoinhibitted $(\beta=0)$, coccolithophores mortality is increased by 0.96 relative to default value;

Results: Prochloroccus overcompetes other small diatoms and haptophytes, coccos are overestimated, coccolithophores still overcompete Phaeocystis, phenology of large diatoms becomes worse.

Prochlorococcus is one of species of prochlorophytes (prokaryotes) type that might be photoinhibited. The changed photoinhibition parameter (varied from 0 to 3 ) did not affect stoichiometry Chl:C ratios but impacted the spatial distribution of 
the PFTs. Figure S4 depicts the December 2003 spatial distribution of prochlorophytes Chla obtained for two experimental runs with different values of the photoinhibition $(\beta)$ parameter. To simulate accurately prochlorophytes, this PFT should be photoinhibited (recommended parameter $\beta$ 1.2)

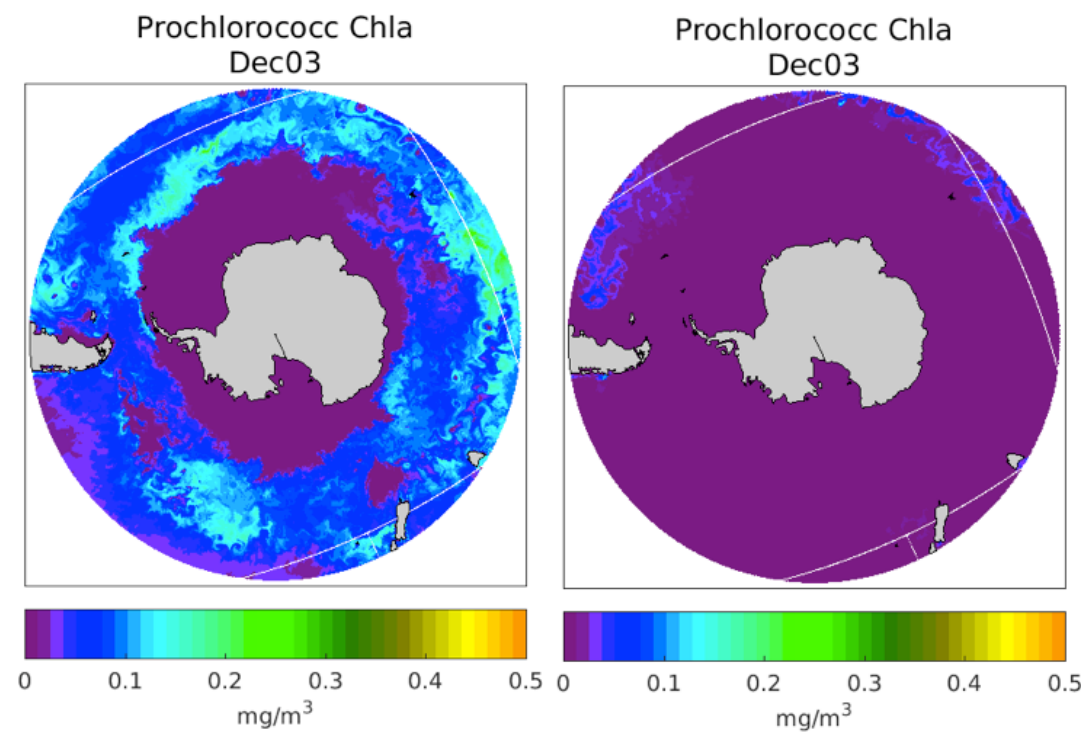

Figure S4. Sensitivity to assumed photoacclimation (photoinhibition parameter): December 2003 prochlorophytes (Prochlorococcus) Chla: $\beta=0$ (left); $\beta=0.9$ (right).

Recommendation: Prochloroccus should be photoinhibited, decrease palatability of "other large", increase size of large diatoms, increase coccolithophores mortality.

Table S4. Assumed size and photosynthesis parameters of the simulated PFTs

\begin{tabular}{lcccccc}
\hline Param $\backslash$ PFTs & diatom & Phaeo & small euk & Prochlor & Nfixer & coccolitho \\
\hline size $(d m)$ & 18 & 5 & 2.2 & 0.6 & 11 & 5 \\
$P_{\text {max }}^{C}$ & 1.79 & 1.67 & 1.73 & 1.09 & 0.31 & 1.11 \\
$k_{\text {sat }_{N}}$ & 0.451 & 0.11 & 0.047 & 0.007 & & 0.079 \\
$k_{\text {sat }_{P}}$ & 0.028 & 0.007 & 0.003 & 0.0004 & 0.076 & 0.005 \\
mfunc & silicified & & silicified & & & calcifier \\
$\beta$ & & & & 1.2 & & \\
\hline
\end{tabular}

Nomenclature as in Table S1. Small eukaryotes are small diatoms

RunB Changes: size of large diatoms $-18 \mu \mathrm{m}$.

Results: Improved large diatom phenology and dominance (more plausible winter distribution, see Figure S5), just slightly improved other large, small diatoms and coccolithophores. 

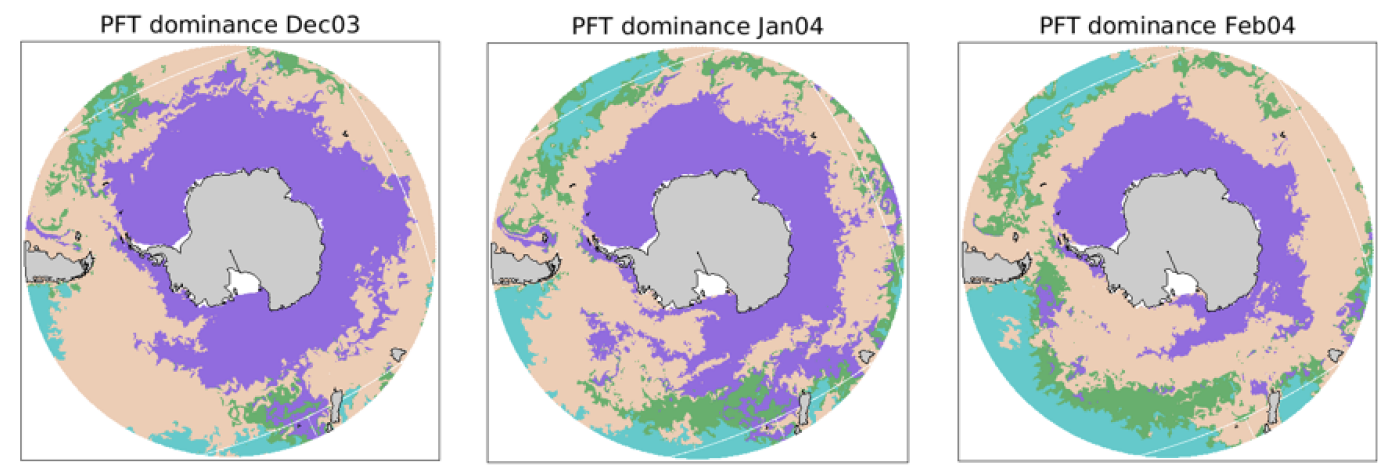

mixed diatom hapto pico

Figure S5. The Southern Ocean PFT dominance simulated with Darwin-MITgcm for December 2003, January 2004 and February 2004.

Recommendation: to keep size of large diatoms - $18 \mu \mathrm{m}$ (Table S4); to increase slightly coccolithophores mortality.
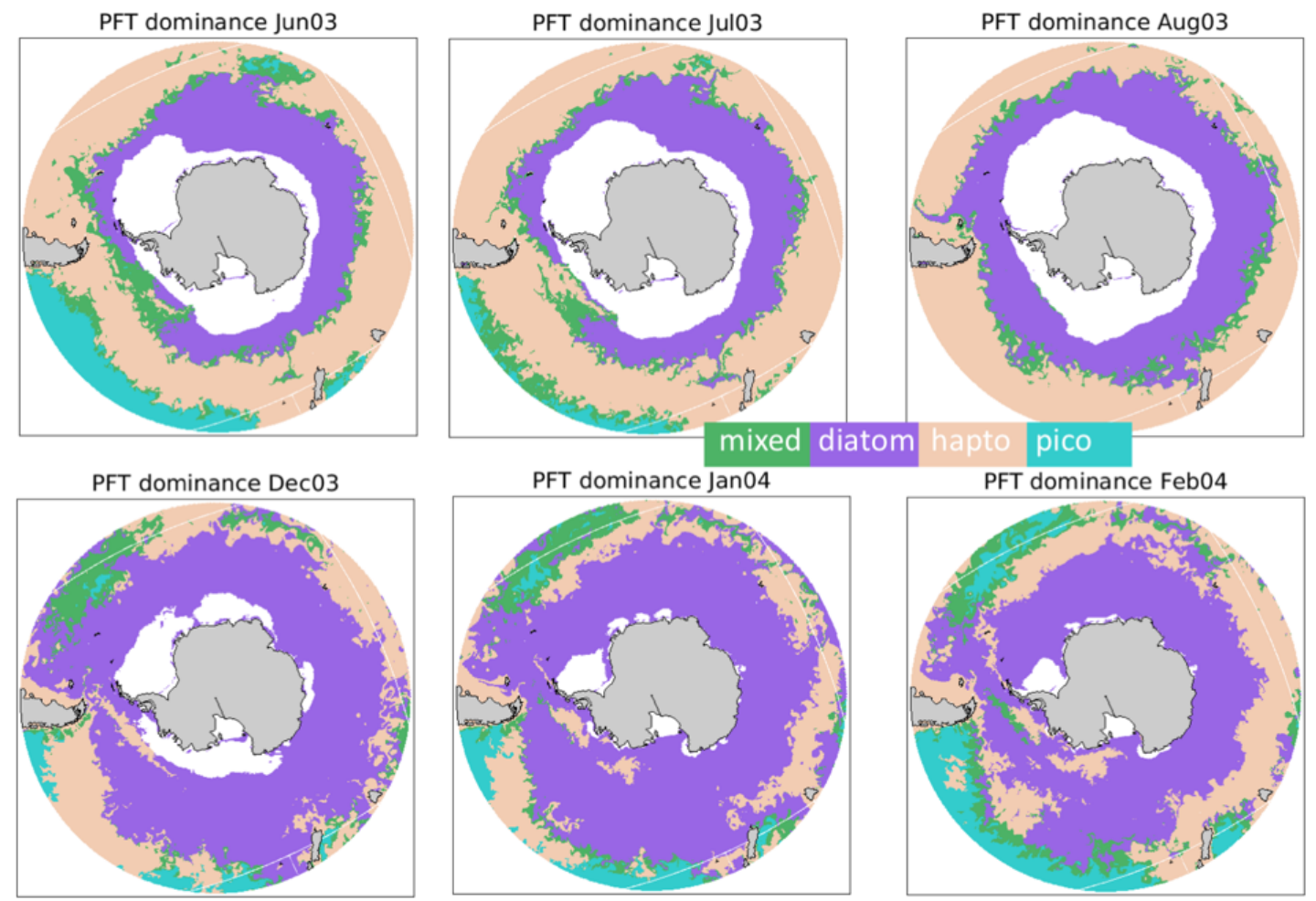

Figure S6. PFT dominance simulated with Darwin-MITgcm for 2003/2004, which is in a good agreement with the PFT dominance provided by PHYSAT satellite data product (http://log.cnrs.fr/Physat-2?lang=fr; Alvain et al. 2008). Pico - represents prochlorophytes. The solution is masked by the area with sea ice concentration $>75 \%$. 
RunC Changes: $r_{j, k=1}=0.76$; coccolithophores mortality increased by $0.95 ; \beta($ Prochlor $)=1.2$; changed for "other pico" $P_{\max }^{C}$ and $k_{\text {sat }}$.

Recommendation: to further adjust $P_{\max }^{C}$ and $k_{\text {sat }}$ for small diatoms; decrease grazing pressure for coccolithophores; decrease "large diatoms" mortality by 1.25 to prolong "large diatom" bloom.

5 Final Results: When introducing two distinct size classes for diatoms (as two different model variables) we are able to improve the spatial distribution and phenology of the simulated diatoms and Phaeocystis ant.: later start and longer duration of the large phytoplankton bloom (Figure S5, S6); patterns of Phaeocystis ant. distribution along the Polar frontal zone in Summer (Alvain et al., 2008; Deppeler and Davidson, 2017).

\section{S2 Hyperspectral satellite-based DOAS coccolithophores fits for the Great Calcite Belt}

10 Figure S7 depicts diatom Chla retrived with PhytoDOAS applied to hyperspectral information measured by the Scanning Imaging Absorption Spectrometer for Atmospheric Chartography (SCIAMACHY) averaged over March 2012 (Bracher et al., 2017). The figure illustrates the sparsity of the information available for the Great Calcite Belt and Chla overestimation in comparison with OC-PFT and SynSenPFT estimates.

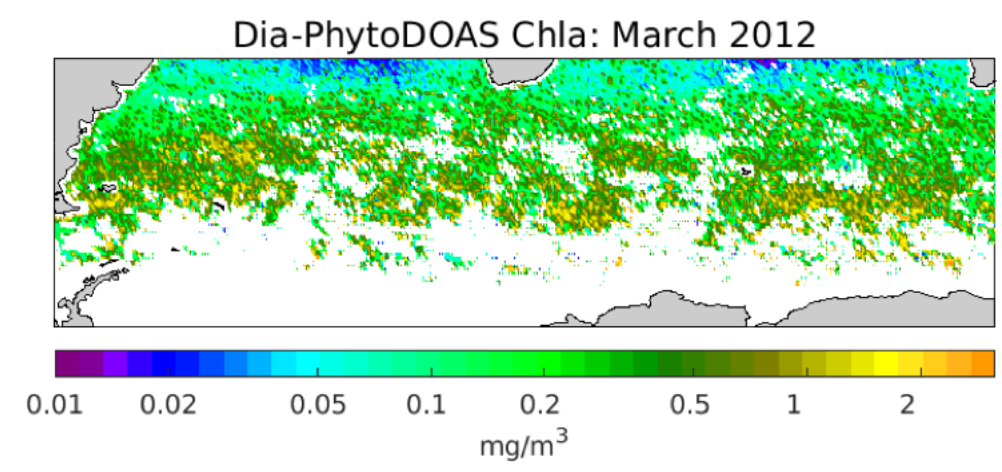

Figure S7. The SCIAMACHY-based PhytoDOAS diatoms Chla retrievals distributed over the domain shown by Smith et al. (2017) for March 2012

\section{S3 PFT seasonal composition}

15 Figure S8 shows seasonal variation of the meridional distribution of zonally averaged phytoplankton composition for four sections of the Southern Ocean. 

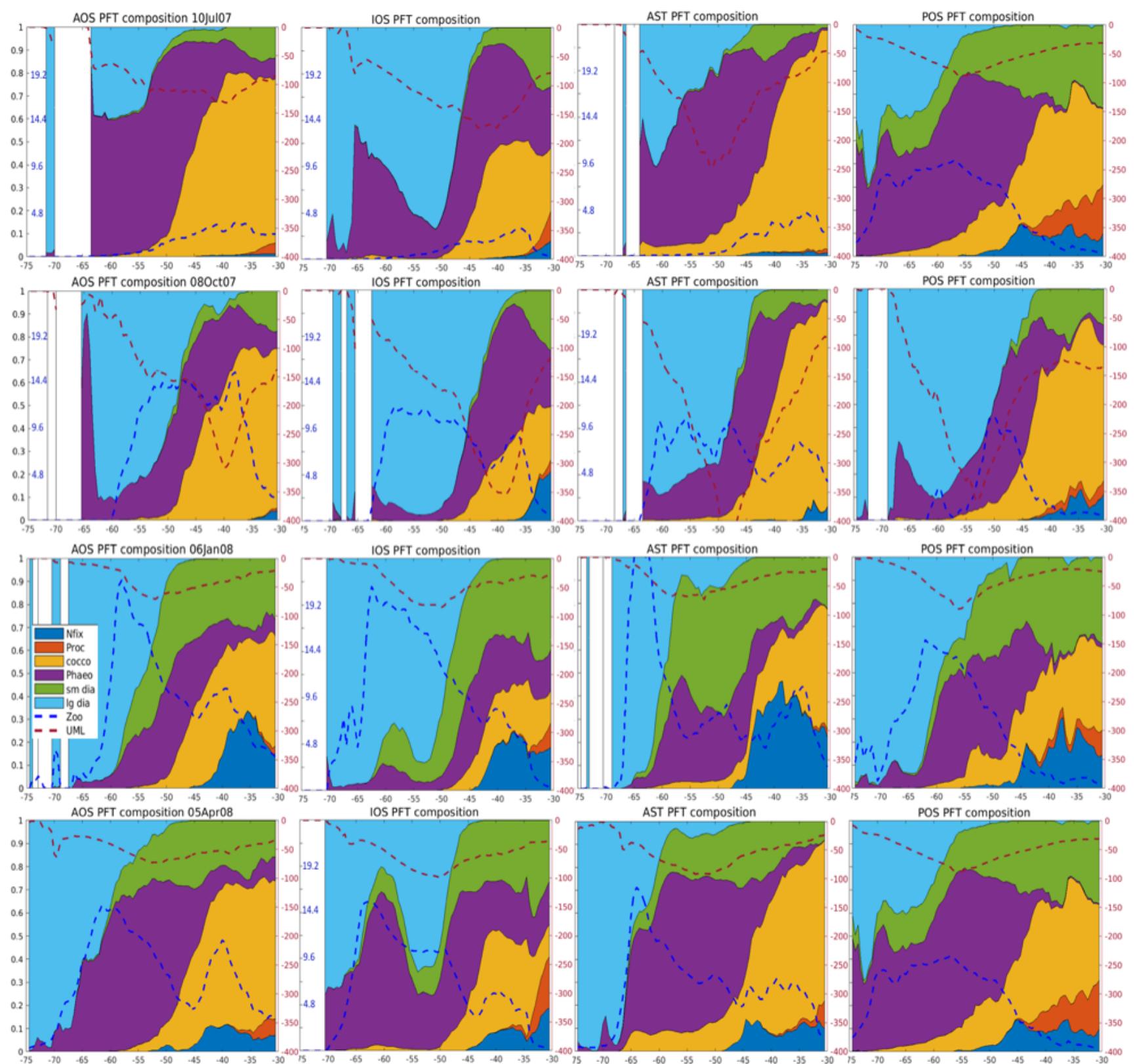

Figure S8. Meridional (from $75^{\circ} \mathrm{S}$ to $30^{\circ} \mathrm{S}$ ) distribution of zonally averaged phytoplankton composition for the Atlantic Ocean sector (AOS, $\left.60^{\circ} \mathrm{W}-18^{\circ} \mathrm{E}\right)$, the Indian Ocean sector (IOS, $\left.18^{\circ} \mathrm{E}-120^{\circ} \mathrm{E}\right)$, Australian sector (AST, $\left.120^{\circ} \mathrm{E}-180^{\circ} \mathrm{E}\right)$ and the Pacific Ocean sector $(\mathrm{POS}$, $180^{\circ} \mathrm{E}-60^{\circ} \mathrm{W}$ ) for experiment PHAEO on 10 July 2007, 8 October 2007, 6 January 2008 and 5 April 2008. The red dashed curve represents the upper mixed layer depth (zonally averaged, $\mathrm{m}$ ). The blue curve represents zonally averaged zooplankton concentration $\left(\mathrm{mgC}^{-3}\right.$ ). 


\section{S4 Phytoplankton traits based on in situ measurements}

Table S5 and S6 review the information on the photophysiological parameters of diatoms and Phaeocystis ant. available from in situ and laboratory observations.

Table S5. Measurement-based diatom photosynthesis parameters

\begin{tabular}{|c|c|c|c|}
\hline Parameter & Units & Parameter value & References \\
\hline \multirow[t]{4}{*}{$P_{\max }^{B}$} & $m g C(m g C h l a)^{-1} h^{-1}$ & $0.44 \pm 0.02-0.54 \pm 0.5$ & Arrigo et al. 2010 \\
\hline & & $0.12 \pm 0.01-0.21 \pm 0.001$ & Kropuenske et al. 2009 \\
\hline & & $0.87 \pm 0.17-1.52 \pm 0.22$ & Kropuenske et al. 2009 \\
\hline & & $1.23 \pm 0.29(5.84 \pm 1.89)$ & Alderkamp et al. 2012 \\
\hline \multirow[t]{3}{*}{$P_{\max }^{C}$} & $d a y^{-1}$ & $0.15 \pm 0.06-0.41 \pm 0.37$ & Arrigo et al. 2010 \\
\hline & & $0.41-0.81$ & Arrigo et al. (2003) \\
\hline & & $0.20 \pm 0.06(0.79 \pm 0.22)$ & Alderkamp et al. 2012 \\
\hline \multirow[t]{3}{*}{$\alpha$} & $m g C(m g C h l a)^{-1} h^{-1}\left(m m o l p h o t o n s m^{-2} s^{-1}\right)^{-1}$ & $0.014 \pm 0.005-0.043 \pm 0.015$ & Arrigo et al. 2010 \\
\hline & & $0.008-0.016$ & Arrigo et al. 2003 \\
\hline & & $0.058 \pm 0.009(0.149 \pm 0.066)$ & Alderkamp et al. 2012 \\
\hline \multirow[t]{4}{*}{ Growth rate $\mu$} & $d a y^{-1}$ & $0.04-0.47$ & Strzepek et al. 2002 \\
\hline & & $0.06 \pm 0.01-0.11 \pm 0.06$ & Arrigo et al. (2010) \\
\hline & & $0.2-1.0$ & Trimborn et al. 2017 \\
\hline & & $0.05 \pm 0.001-0.16 \pm 0.46$ & Alderkamp et al. (2012) \\
\hline$\mu: \mu_{\max }$ & & $0.25-1.0$ & Strzepek et al. 2012 \\
\hline \multirow[t]{3}{*}{$a^{*}$} & $m^{2} m g C h l a^{-1}$ & $0.006-0.011$ & Arrigo et al. 2003 \\
\hline & & $0.012 \pm 0.002-0.014 \pm 0.002$ & Arrigo et al. 2010 \\
\hline & & $0.027 \pm 0.003-0.031 \pm 0.008$ & Alderkamp et al. 2012 \\
\hline
\end{tabular}


Table S6. Measurement-based Phaeocystis ant. photosynthesis parameters

\begin{tabular}{|c|c|c|c|}
\hline Parameter & Units & $\begin{array}{l}\text { Parameter value } \\
\text { solitary (colonial) }\end{array}$ & References \\
\hline \multirow[t]{8}{*}{$P_{\max }^{B}$} & $m g C(m g C h l a)^{-1} h^{-1}$ & $1.1-3.2(0.5-3)$ & Shields and Smith 2009 \\
\hline & & $0.3-0.55(0.1-1.45)$ & \\
\hline & & decrease with increase of & \\
\hline & & percentage of colonial & \\
\hline & & $1.4 \pm 0.3-6.4 \pm 0.3$ & Arrigo et al. 2010 \\
\hline & & $1.16 \pm 0.08-1.54 \pm 0.06$ & Kropuenske et al. 2009 \\
\hline & & $0.79 \pm 0.56-3.07 \pm 0.64$ & Kropuenske et al. 2009 \\
\hline & & $4.63 \pm 0.43(7.77 \pm 0.71)$ & Alderkamp et al. 2012 \\
\hline \multirow[t]{2}{*}{$P_{\max }^{C}$} & $d a y^{-1}$ & $0.57 \pm 0.08-0.85 \pm 0.23$ & Arrigo et al. 2010 \\
\hline & & $0.63 \pm 0.1(1.15 \pm 0.20)$ & Alderkamp et al. 2012 \\
\hline \multirow[t]{3}{*}{$\alpha$} & $m g C(m g C h l a)^{-1} h^{-1} \ldots$ & $0.01-0.08(0.01-0.17)$ & Shields and Smith 2009 \\
\hline & $\left(\text { mmolphotonsm }^{-2} s^{-1}\right)^{-1}$ & $0.038 \pm 0.008-0.11 \pm 0.019$ & Arrigo et al. 2010 \\
\hline & & $0.062 \pm 0.01(0.136 \pm 0.01)$ & Alderkamp et al. 2012 \\
\hline \multirow[t]{10}{*}{ Growth rate $\mu$} & $d a y^{-1}$ & $0.13(0.38)$ & Shields and Smith 2009 \\
\hline & & $0.15-0.7$ & Luxem et al. 2017 \\
\hline & & $0.49(0.55)$ & van Leeuwe and Stefels 2007 \\
\hline & & $0.20-0.53$ & Strzepek et al. 2011, 2012 \\
\hline & & $0.07 \pm 0.01-0.35 \pm 0.13$ & Arrigo et al. 2010 \\
\hline & & 0.17 & Tang et al. 2009 \\
\hline & & 0.41 & Walker O. Smith 1999 \\
\hline & & $0.21-0.27$ & Sedwick et al. 2007 \\
\hline & & $0.35-0.48$ & Trimborn et al. 2017 \\
\hline & & $0.19 \pm 0.006(0.38 \pm 0.025)$ & Alderkamp et al. 2012 \\
\hline$\mu: \mu_{\max }$ & & $0.54-1.0$ & Strzepek et al. 2011 \\
\hline \multirow[t]{4}{*}{ Specific growth rate } & $d a y^{-1}$ & $0.05-0.4$ & Moisan and Mitchell 2018 \\
\hline & & $\mathrm{T}-1.5 \circ C$ to $4 \circ C$ & \\
\hline & & at low $\mathrm{T}$ photoinhibit & \\
\hline & & after $100\left(\right.$ mmolquantam $\left.^{-2} s^{-1}\right)$ & \\
\hline \multirow[t]{3}{*}{$a^{*}$} & $m^{2} m g C h l a^{-1}$ & $0.009-0.0181$ & Arrigo et al. 2010 \\
\hline & & $0.057 \pm 0.023(0.030 \pm 0.022)$ & van Leeuwe and Stefels 2007 \\
\hline & & $0.024 \pm 0.002(0.029 \pm 0.0041)$ & Alderkamp et al. 2012 \\
\hline
\end{tabular}




\section{S5 Additional information on model evaluation against in situ}

There are three video files additionally provided via AV-Portal of the German National Library of Science and Technology (TIB, Hannover): https://doi.org/10.5446/42871; https://doi.org/10.5446/42873; https://doi.org/10.5446/42872. The movies depict distribution of diatoms, haptophytes and prokaryotes Chla for model snapshots over the time period from August 2002 to April

5 2012. These simulated Chla distributions are shown with in situ observations available within the time window \pm 1 weeks around the date of the snapshot. Table S7 and S8 present the statistics of goodness of model to data fit for log-transformed diatoms and haptophytes Chla. Table S9 shows the statistics with respect to physical values of Chla at specific sections of Longhurst's bgc provinces. Figures S9 - S11 show frequency distributions of observed and simulated Chla as well as their differences for diatom, haptophytes and prokaryotes, respectively.

Table S7. Diatom Chla: model vs. in situ log-transformed statistics at Longhurst's provinces

\begin{tabular}{lcccccccc}
\hline criteria $\backslash$ bioms & APLR & ANTA & SANT & SSTC & FKLD & EAFR & AUSW & AUSE \\
\hline MAE & 0.65 & 0.62 & 0.54 & 0.48 & 0.40 & 0.72 & 0.27 & 0.29 \\
RMSE & 0.82 & 0.75 & 0.64 & 0.55 & 0.48 & 0.85 & 0.33 & 0.39 \\
RMSE unbiased & 0.82 & 0.59 & 0.64 & 0.45 & 0.45 & 0.82 & 0.33 & 0.39 \\
bias & -0.004 & 0.45 & 0.01 & 0.32 & -0.17 & 0.20 & -0.08 & -0.06 \\
\hline
\end{tabular}

MAE - mean absolute error; RMSE — root mean squared error; bioms are the Longhurst's biogeochemical provinces Longhurst (1998): Austral Polar Province (APLR), Antarctic Province (ANTA), Subantarctic Water Ring Province (SANT), South Subtropical Convergence Province (SSTC), Southwest Atlantic Shelves Province (FKLD), Eastern Africa Coastal Province (EAFR), Australia-Indonesia Coastal Province (AUSW), East Australian Coastal Province (AUSE).

Table S8. Haptophytes Chla: model vs. in situ log-transformed statistics at Longhurst's provinces

\begin{tabular}{lcccccccc}
\hline criteria $\backslash$ bioms & APLR & ANTA & SANT & SSTC & FKLD & EAFR & AUSW & AUSE \\
\hline MAE & 0.43 & 0.58 & 0.32 & 0.25 & 0.22 & 0.96 & 0.56 & 1.01 \\
RMSE & 0.56 & 0.67 & 0.39 & 0.34 & 0.28 & 1.01 & 0.67 & 1.06 \\
RMSE unbiased & 0.50 & 0.64 & 0.39 & 0.34 & 0.26 & 0.32 & 0.39 & 0.30 \\
bias & 0.25 & 0.21 & 0.08 & 0.00 & -0.11 & 0.96 & 0.54 & 1.01 \\
\hline
\end{tabular}

Same as in Table S7 
Table S9. Diatom|haptophytes: model vs. in situ statistics $\left(\mathrm{mgChlam}^{-3}\right)$ for specific sections of Longhurst's provinces

\begin{tabular}{|c|c|c|c|c|c|}
\hline bioms $\backslash$ criteria & MAE & RMSE & RMSE unbiased & bias & $\mathrm{N}$ \\
\hline APLR 1$] 180^{\circ} \mathrm{W}-150^{\circ} \mathrm{W}[$ & $0.66 \mid 0.19$ & $1.41 \mid 0.26$ & $1.41 \mid 0.20$ & $-0.08 \mid 0.17$ & $54 \mid 53$ \\
\hline APLR $2\left[150^{\circ} \mathrm{W}-110^{\circ} \mathrm{W}[\right.$ & $0.81 \mid 0.08$ & $1.30 \mid 0.11$ & $1.18 \mid 0.11$ & $-0.5 \mid 0.02$ & $40 \mid 40$ \\
\hline APLR3 $\left[110^{\circ} \mathrm{W}-65^{\circ} \mathrm{W}[\right.$ & $1.13 \mid 0.21$ & $2.13 \mid 0.41$ & $2.08 \mid 0.39$ & $-0.47 \mid 0.12$ & $803 \mid 812$ \\
\hline APLR $1-3\left[180^{\circ} \mathrm{W}-65^{\circ} \mathrm{W}[\right.$ & 1.1 & $2.07 \mid$ & $2.02 \mid$ & -0.45 & $897 \mid$ \\
\hline APLR4 $\left[65^{\circ} \mathrm{W}-25^{\circ} \mathrm{W}[\right.$ & $0.45 \mid 0.34$ & $0.95|0.580 .93| 0.58$ & $-0.17 \mid 0.03$ & $293 \mid 295$ & \\
\hline APLR5 $\left[25^{\circ} \mathrm{W}-110^{\circ} \mathrm{E}[\right.$ & $1.45 \mid 0.27$ & $1.97 \mid 0.51$ & $1.57 \mid 0.50$ & $1.19 \mid-0.08$ & $64 \mid 64$ \\
\hline APLR6 $\left[110^{\circ} \mathrm{E}-155^{\circ} \mathrm{E}[\right.$ & & & & & $\mathrm{NaN} \mid \mathrm{NaN}$ \\
\hline APLR7 $\left[155^{\circ} \mathrm{E}-180^{\circ} \mathrm{E}[\right.$ & $2.87 \mid$ & 3.20 & 3.19 & 0.16 & $33 \mid \mathrm{NaN}$ \\
\hline APLR5-7 $\left[25^{\circ} \mathrm{E}-180^{\circ} \mathrm{E}[\right.$ & $1.91 \mid$ & 2.46 & $2.21 \mid$ & $0.84 \mid$ & $97 \mid$ \\
\hline ANTA1 $] 180^{\circ} \mathrm{W}-150^{\circ} \mathrm{W}[$ & $0.88 \mid 0.07$ & $0.92 \mid 0.08$ & $0.28 \mid 0.08$ & $0.88 \mid-0.02$ & $26 \mid 22$ \\
\hline ANTA $2\left[150^{\circ} \mathrm{W}-110^{\circ} \mathrm{W}[\right.$ & $0.20 \mid 0.34$ & $0.23 \mid 0.34$ & $0.12 \mid 0.06$ & $0.19 \mid 0.34$ & $29 \mid 29$ \\
\hline ANTA3 $\left[110^{\circ} \mathrm{W}-65^{\circ} \mathrm{W}[\right.$ & $0.19 \mid 0.23$ & $0.24 \mid 0.25$ & $0.19 \mid 0.11$ & $0.14 \mid 0.23$ & $45 \mid 46$ \\
\hline ANTA $1-3\left[180^{\circ} \mathrm{W}-65^{\circ} \mathrm{W}[\right.$ & $0.37 \mid$ & $0.51 \mid$ & 0.38 & 0.35 & $100 \mid$ \\
\hline ANTA4 $\left[65^{\circ} \mathrm{W}-25^{\circ} \mathrm{E}[\right.$ & $1.48 \mid 0.22$ & $2.41 \mid 0.27$ & $2.26 \mid 0.26$ & $0.84 \mid-0.09$ & $69 \mid 67$ \\
\hline ANTA5 $\left[25^{\circ} \mathrm{W}-110^{\circ} \mathrm{E}[\right.$ & $0.62 \mid 0.19$ & $1.04 \mid 0.30$ & $0.91 \mid 0.26$ & $0.51 \mid 0.16$ & $55 \mid 54$ \\
\hline ANTA6 $\left[110^{\circ} \mathrm{E}-155^{\circ} \mathrm{E}[\right.$ & $0.14 \mid 0.22$ & $0.15 \mid 0.23$ & $0.04 \mid 0.06$ & $0.14 \mid-0.22$ & $10 \mid 10$ \\
\hline ANTA7 $\left[155^{\circ} \mathrm{E}-180^{\circ} \mathrm{E}[\right.$ & 0.78 & $1.01 \mid$ & 0.78 & 0.66 & $2 \mid$ \\
\hline ANTA5-7 $\left[25^{\circ} \mathrm{E}-180^{\circ} \mathrm{E}[\right.$ & 0.55 & 0.96 & 0.84 & 0.46 & $67 \mid$ \\
\hline SANT1-3 $\left[180^{\circ} \mathrm{W}-65^{\circ} \mathrm{W}[\right.$ & $0.26 \mid 0.23$ & $0.32 \mid 0.28$ & $0.19 \mid 0.17$ & $0.26 \mid 0.23$ & $9 \mid 13$ \\
\hline SANT4 $\left[65^{\circ} \mathrm{W}-25^{\circ} \mathrm{W}[\right.$ & $0.28 \mid 0.13$ & $0.43 \mid 0.18$ & $0.37 \mid 0.17$ & $-0.21 \mid 0.05$ & $316 \mid 330$ \\
\hline SANT5 $\left[25^{\circ} \mathrm{W}-110^{\circ} \mathrm{E}[\right.$ & $0.36 \mid 0.10$ & $0.41 \mid 0.11$ & $0.34 \mid 0.07$ & $0.23 \mid 0.09$ & $40 \mid 39$ \\
\hline SANT6 $\left[110^{\circ} \mathrm{E}-155^{\circ} \mathrm{E}[\right.$ & $0.12 \mid 0.32$ & $0.13 \mid 0.36$ & $0.05 \mid 0.22$ & $0.12 \mid-0.23$ & $37 \mid 55$ \\
\hline SANT5-7 $\left[25^{\circ} \mathrm{E}-155^{\circ} \mathrm{E}[\right.$ & 0.24 & $0.31 \mid$ & 0.26 & 0.18 & $77 \mid$ \\
\hline SSTC4 $\left[65^{\circ} \mathrm{W}-25^{\circ} \mathrm{W}[\right.$ & $0.09 \mid 0.14$ & $0.14 \mid 0.28$ & $0.14 \mid 0.28$ & $0 \mid-0.05$ & $72 \mid 1065$ \\
\hline $\operatorname{SSTC} 5\left[25^{\circ} \mathrm{W}-110^{\circ} \mathrm{E}[\right.$ & $0.05 \mid 0.02$ & $0.05 \mid 0.03$ & $0.04 \mid 0.03$ & $0.04 \mid 0.01$ & $7 \mid 8$ \\
\hline SSTC6 $\left[110^{\circ} \mathrm{E}-155^{\circ} \mathrm{E}[\right.$ & $0.05 \mid 0.17$ & $0.07 \mid 0.23$ & $0.07 \mid 0.23$ & $0.01 \mid 0.01$ & $23 \mid 40$ \\
\hline $\operatorname{SSTC} 5-7\left[25^{\circ} \mathrm{E}-155^{\circ} \mathrm{E}[\right.$ & 0.05 & 0.06 & 0.06 & $0.02 \mid$ & $30 \mid$ \\
\hline
\end{tabular}




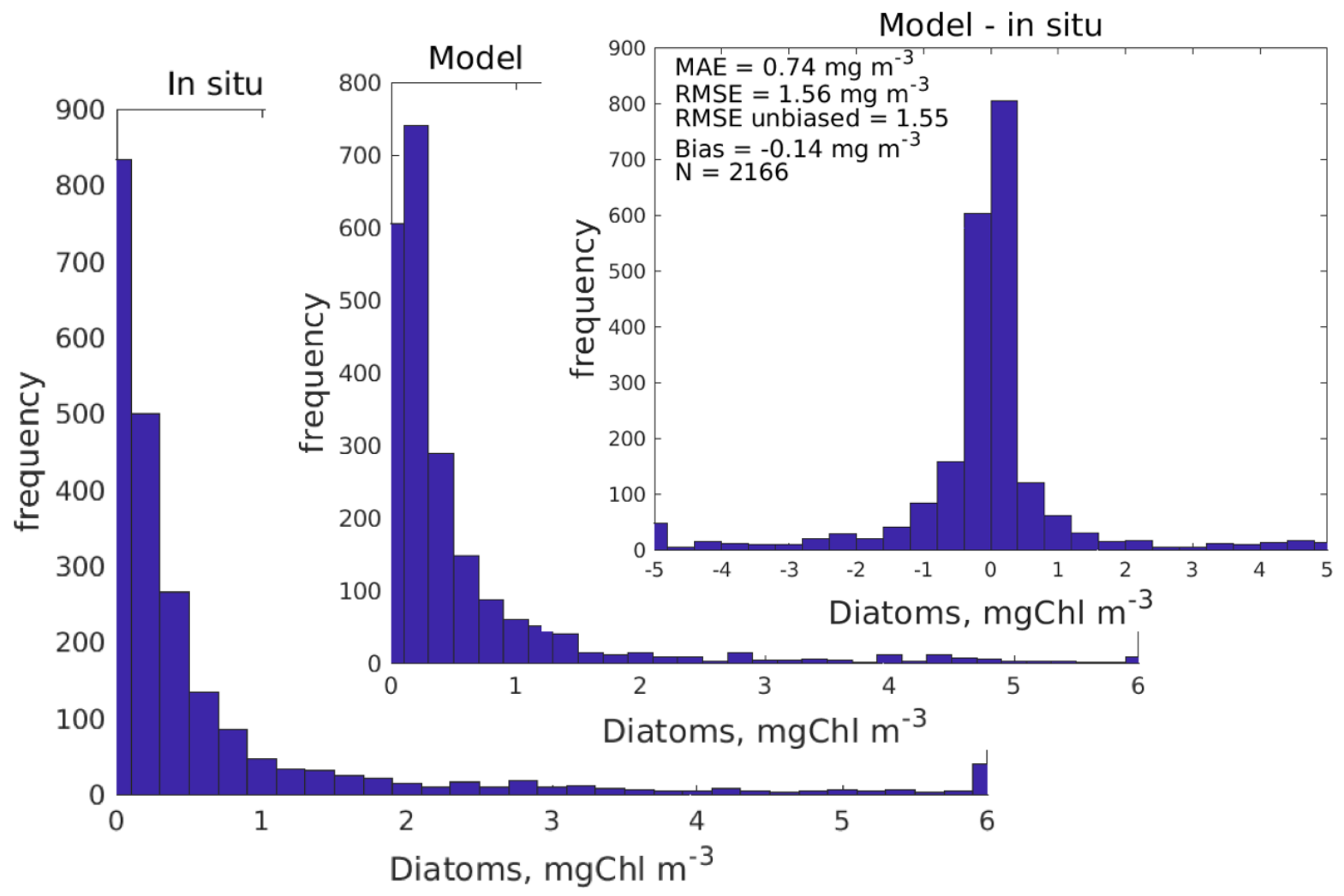

Figure S9. The frequency distribution of diatom Chla based on the in situ observations and model simulation, and frequency distribution of differences between model and observations. 


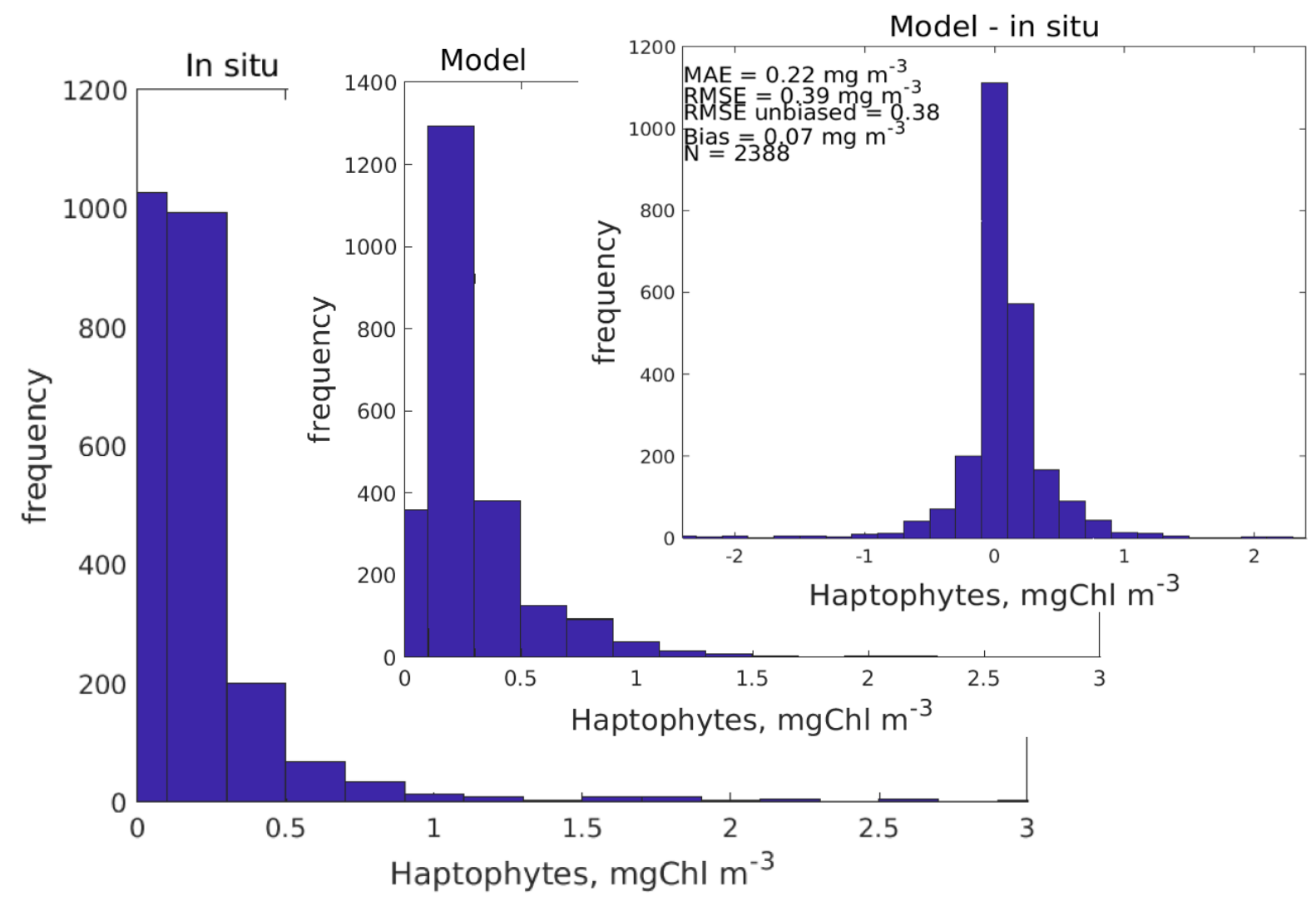

Figure S10. Same as Figure S9 but for haptophytes. 


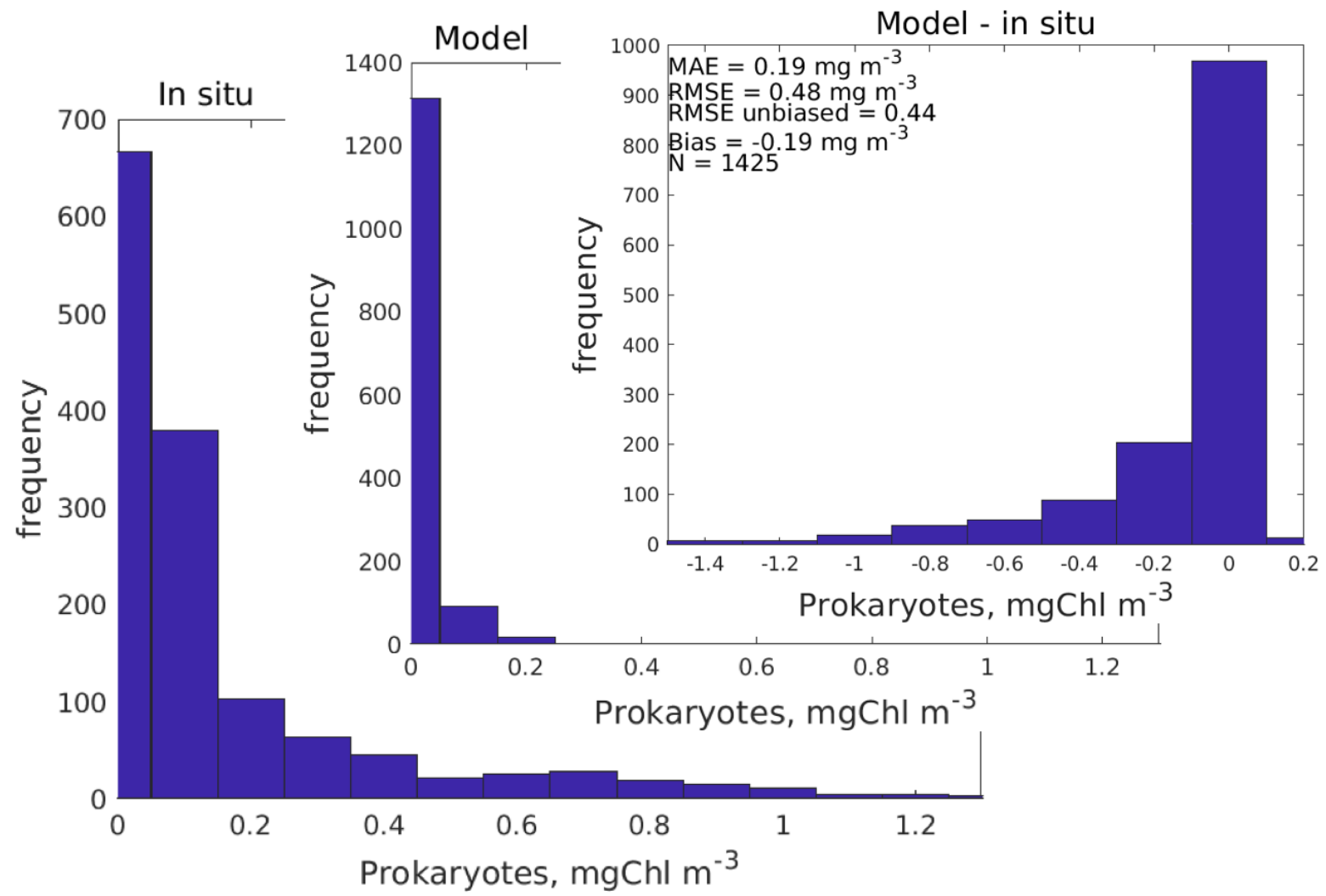

Figure S11. Same as Figures S9 and S10 but for pico-phytoplankton (prokaryotes). 


\section{References}

Alderkamp, A.-C., Kulk, G., Buma, A. G. J., Visser, R. J. W., Van Dijken, G. L., Mills, M. M., and Arrigo, K. R.: THE EFFECT OF IRON LIMITATION ON THE PHOTOPHYSIOLOGY OF PHAEOCYSTIS ANTARCTICA (PRYMNESIOPHYCEAE) AND FRAGILARIOPSIS CYLINDRUS (BACILLARIOPHYCEAE) UNDER DYNAMIC IRRADIANCE1, Journal of Phycology, 48, 45-59, https://doi.org/10.1111/j.1529-8817.2011.01098.x, 2012.

Alvain, S., Moulin, C., Dandonneau, Y., and Loisel, H.: Seasonal distribution and succession of dominant phytoplankton groups in the global ocean: A satellite view, Global Biogeochemical Cycles, 22, https://doi.org/10.1029/2007GB003154, 2008.

Arrigo, K. R., Robinson, D. H., Dunbar, R. B., Leventer, A. R., and Lizotte, M. P.: Physical control of chlorophyll a, POC, and TPN distributions in the pack ice of the Ross Sea, Antarctica, Journal of Geophysical Research: Oceans, 108, https://doi.org/10.1029/2001JC001138, 2003.

Arrigo, K. R., Mills, M. M., Kropuenske, L. R., van Dijken, G. L., Alderkamp, A.-C., and Robinson, D. H.: Photophysiology in Two Major Southern Ocean Phytoplankton Taxa: Photosynthesis and Growth of Phaeocystis antarctica and Fragilariopsis cylindrus under Different Irradiance Levels, Integrative and Comparative Biology, 50, 950-966, https://doi.org/10.1093/icb/icq021, 2010.

Bracher, A., Dinter, T., Wolanin, A., Rozanov, V. V., Losa, S., and Soppa, M. A.: Global monthly mean chlorophyll a surface concentrations from August 2002 to April 2012 for diatoms, coccolithophores and cyanobacteria from PhytoDOAS algorithm version 3.3 applied to SCIAMACHY data, link to NetCDF files in ZIP archive, https://doi.org/10.1594/PANGAEA.870486, https://doi.org/10.1594/PANGAEA. 870486, in supplement to: Losa, Svetlana; Soppa, Mariana A; Dinter, Tilman; Wolanin, Aleksandra; Brewin, Robert J W; Bricaud, Annick; Oelker, Julia; Peeken, Ilka; Gentili, Bernard; Rozanov, Vladimir V; Bracher, Astrid (2017): Synergistic Exploitation of Hyper- and MultiSpectral Precursor Sentinel Measurements to Determine Phytoplankton Functional Types (SynSenPFT). Frontiers in Marine Science, 4(203), 22 pp, https://doi.org/10.3389/fmars.2017.00203, 2017.

Deppeler, S. L. and Davidson, A. T.: Southern Ocean Phytoplankton in a Changing Climate, Frontiers in Marine Science, 4, 40, https://doi.org/10.3389/fmars.2017.00040, 2017.

Dutkiewicz, S., Hickman, A. E., Jahn, O., Gregg, W. W., Mouw, C. B., and Follows, M. J.: Capturing optically important constituents and properties in a marine biogeochemical and ecosystem model, Biogeosciences, 12, 4447-4481, https://doi.org/10.5194/bg-12-4447-2015, 2015.

Kropuenske, L. R., Mills, M. M., van Dijken, G. L., Bailey, S., Robinson, D. H., Welschmeyer, N. A., and Arrigoa, K. R.: Photophysiology in two major Southern Ocean phytoplankton taxa: Photoprotection in Phaeocystis antarctica and Fragilariopsis cylindrus, Limnology and Oceanography, 54, 1176-1196, https://doi.org/10.4319/lo.2009.54.4.1176, 2009.

Longhurst, A.: Ecological Geography of the Sea, Academic press, 1998.

Luxem, K. E., Ellwood, M. J., and Strzepek, R. F.: Intraspecific variability in Phaeocystis antarctica's response to iron and light stress, PLOS ONE, 12, 1-14, https://doi.org/10.1371/journal.pone.0179751, 2017.

Moisan, T. A. and Mitchell, B. G.: Modeling Net Growth of Phaeocystis antarctica Based on Physiological and Optical Responses to Light and Temperature Co-limitation, Frontiers in Marine Science, 4, 437, https://doi.org/10.3389/fmars.2017.00437, 2018.

Sedwick, P. N., Garcia, N. S., Riseman, S. F., Marsay, C. M., and DiTullio, G. R.: Evidence for high iron requirements of colonial Phaeocystis antarctica at low irradiance, Biogeochemistry, 83, 83-97, https://doi.org/10.1007/s10533-007-9081-7, 2007.

Shields, A. R. and Smith, W. O.: Size-fractionated photosynthesis/irradiance relationships during Phaeocystis antarctica-dominated blooms in the Ross Sea, Antarctica, Journal of Plankton Research, 31, 701-712, https://doi.org/10.1093/plankt/fbp022, 2009. 
Smith, H. E. K., Poulton, A. J., Garley, R., Hopkins, J., Lubelczyk, L. C., Drapeau, D. T., Rauschenberg, S., Twining, B. S., Bates, N. R., and Balch, W. M.: The influence of environmental variability on the biogeography of coccolithophores and diatoms in the Great Calcite Belt, Biogeosciences, 14, 4905-4925, https://doi.org/10.5194/bg-14-4905-2017, 2017.

Soppa, M. A., Völker, C., and Bracher, A.: Diatom Phenology in the Southern Ocean: Mean Patterns, Trends and the Role of Climate Oscillations, Remote Sensing, 8, https://doi.org/10.3390/rs8050420, 2016.

Strzepek, R. F., Maldonado, M. T., Hunter, K. A., Frew, R. D., and Boyd, P. W.: Adaptive strategies by Southern Ocean phytoplankton to lessen iron limitation: Uptake of organically complexed iron and reduced cellular iron requirements, Limnology and Oceanography, 56, 1983-2002, https://doi.org/10.4319/lo.2011.56.6.1983, 2011.

Strzepek, R. F., Hunter, K. A., Frew, R. D., Harrison, P. J., and Boyd, P. W.: Iron-light interactions differ in Southern Ocean phytoplankton, Limnology and Oceanography, 57, 1182-1200, https://doi.org/10.4319/lo.2012.57.4.1182, 2012.

Tang, K. W., Smith, W. O., Shields, A. R., and Elliott, D. T.: Survival and recovery of <i>Phaeocystis antarctica</i> (Prymnesiophyceae) from prolonged darkness and freezing, Proceedings of the Royal Society B: Biological Sciences, 276, 81-90, https://doi.org/10.1098/rspb.2008.0598, 2009.

Trimborn, S., Hoppe, C. J., Taylor, B. B., Bracher, A., and Hassler, C.: Physiological characteristics of open ocean and coastal phytoplankton communities of Western Antarctic Peninsula and Drake Passage waters, Deep Sea Research Part I: Oceanographic Research Papers, 98, 115 - 124, https://doi.org/10.1016/j.dsr.2014.12.010, 2015.

Trimborn, S., Thoms, S., Brenneis, T., Heiden, J. P., Beszteri, S., and Bischof, K.: Two Southern Ocean diatoms are more sensitive to ocean acidification and changes in irradiance than the prymnesiophyte Phaeocystis antarctica, Physiologia Plantarum, 160, 155-170, https://doi.org/10.1111/ppl.12539, 2017.

van Leeuwe, M. A. and Stefels, J.: Photosynthetic responses in Phaeocystis antarctica towards varying light and iron conditions, Biogeochemistry, 83, 61-70, https://doi.org/10.1007/s10533-007-9083-5, 2007.

Walker O. Smith, Jr, D. M. S. M.: Phytoplankton growth rates in the Ross Sea, Antarctica, determined by independent methods: temporal variations, Journal of Plankton Research, 21, 1519-1536, https://doi.org/10.1093/plankt/21.8.1519, 1999.

Ward, B. A., Marañón, E., Sauterey, B., Rault, J., and Claessen, D.: The Size Dependence of Phytoplankton Growth Rates: A Trade-Off between Nutrient Uptake and Metabolism, The American Naturalist, 189, 170-177, https://doi.org/10.1086/689992, 2017. 